\title{
Editorial Note: Intelligent Systems for Digital Archiving, Analysis \& Documentation
}

(C) Springer Science+Business Media, LLC, part of Springer Nature 2017

Multimedia Tools and Applications gratefully acknowledges the editorial work of the scholars listed below on the special issue entitled, "Intelligent Systems for Digital Archiving, Analysis \& Documentation."

\section{Mikołaj Leszczuk (Corresponding Guest Editor)}

AGH University of Science and Technology, Kraków, Poland

Email: leszczuk@agh.edu.pl

\section{Andrzej Dziech}

AGH University of Science and Technology, Kraków, Poland

Email: dziech@kt.agh.edu.pl

\section{Remigiusz Baran}

Kielce University of Technology, Kielce, Poland

Email: r.baran@tu.kielce.pl 